\title{
Analysis of Nonlinear Vibration Response of Bolt Fixed Cantilever Beam under Harmonic Excitation
}

\author{
Yaobin $\mathrm{Li}^{1,2, *}$, Huadong $\mathrm{Yu}^{1}$, Qianglong Wang ${ }^{2}$, Yang Song ${ }^{2,3}$ and Zhenyu $\mathrm{Liu}^{2}$ \\ ${ }^{1}$ College of Mechanical and Electric Engineering, Changchun University of Science and Technology, Institute of Optics, \\ Changchun 130022, China \\ ${ }^{2}$ Changchun Institute of Optics, Fine Mechanics and Physics Chinese Academy of Sciences, Changchun 130033, China \\ ${ }^{3}$ University of Chinese Academy of Science, Beijing, 100049, China \\ ${ }^{*}$ Corresponding author
}

\begin{abstract}
The local nonlinearity introduced by the bolt assembly are one of the major reasons for the complex dynamics response characteristics of the photoelectric measurement and control equipment. The single-bolt fixed cantilever beam model is adopted to analyze the super harmonic nonlinear response of structure induced by the local nonlinearity in bolt joint connection structure which is widely used in mechanical structures. The implicit dynamic simulation method is utilized to analyze the phase trajectory curve. The amplitude of superharmonic response is more important than the amplitude of forced excitation under excitation frequency of $1 / 2$ times the resonance frequency. The system exhibits quasi-periodic motion and resonance frequencies exists harmonic components under forced excitation of specific frequency load.
\end{abstract}

Keywords-nonlinear vibration; bolt joint; harmonic response

\section{INTRODUCTION}

The simulation result is not correct when using linear dynamic simulation method while nonlinear boundary conditions due to bolt-connected and shaft-axis exist ${ }^{[1]}$. Structural dynamics response changed due to local nonlinearity induced by bolt connection. The influence of the local nonlinearity on the dynamic response of the structure is usually analyzed by means of indirect reconstruction of parameters using either Iwan ${ }^{[2]}$ model which depend on spring and damped element to characterize the stick-slip phenomenon or Force-State Mapping method ${ }^{[3-5]}$. The Nonlinear term in the dynamics equation which is induced by restoring force and damping of the bolted joint interfere influenced the dynamics response and stability of structure. The motion state may change from periodic to quasi-periodic state due to small difference about the sensitive parameter ${ }^{[6]}$. The resonant noise response caused by the non-servo frequency band excitation which comes from strong structural nonlinearity of photoelectric measurement affects the tracking stability and accuracy of the optical system ${ }^{[7-9]}$.

It is necessary to further analyze and simulate the structure nonlinear resonance response noise, so as to provide the design parameters and the theoretical basis for the development of the photoelectric measurement and control equipment with high sensitivity and higher tracking accuracy.

\section{CHARACTERIZATION AND RESONANCE THEORY OF STRUCTURAL NONLINEAR SYSTEM}

The equation of motion of the system with nonlinear excitation under harmonic excitation can be expressed as follows[10]:

$$
\begin{gathered}
M \ddot{x}+C \dot{x}+K x+f_{d}(\dot{x})+f_{s}(x)=F \sin \left(\omega_{0} t+\phi\right) \\
f_{d}(\dot{x})=c_{2} \dot{x}^{2} \\
f_{s}(x)=\alpha x^{2}+\beta x^{3}+\gamma x^{4}
\end{gathered}
$$

where $\mathrm{M}$ is the mass matrix, $\mathrm{K}$ is the damping matrix and $\mathrm{C}$ is the stiffness matrix of the system; $f_{d}(\dot{x})$ is the nonlinear damping term; $f_{s}(x)$ is the nonlinear restoring force for the system. Equation 2 means the common quadratic damping term and Equation 3 shows the common stiffness expression. The $\alpha 、 \beta$ and $\gamma$ are the nonlinear coefficient. The system mar occur super-harmonic response when the external excitation frequency can be written as follows:

$$
\Omega \approx \omega_{f} / n
$$

where $\omega_{0}$ is the system natural frequency and $n$ is the nonpositive integer[10].

\section{DEFINITION OF STRONG LOCAL NONLINEARITY LOW PRETENSION MODEL}

Sketches of the simulated cantilever beam are presented in Figure 1. The deformation of bottom edge of the base is constrained. Three contact elements groups are defined between cantilever beam with base and nut. Bolt and nuts are meshed using 3D-Bolt element (regular hexahedron elements) to ensure proper mesh mapping of the contact surface between contact areas. The deformation of the structure is calculated under the condition of large strain and small strain. The $\mathrm{Z}$ direction of the FEM model is defined as the bolt center axis direction. $\mathrm{X}$ direction is along the length direction of the cantilever beam and the $\mathrm{Y}$ direction is determined according to the right hand screw rule. The load excitation direction is parallel to the bolt central axis and Point $\mathrm{A}$ in Figure 2 is the 
applied point. Meshing conditions are shown in Table.1. External loading and contact definition and calculating

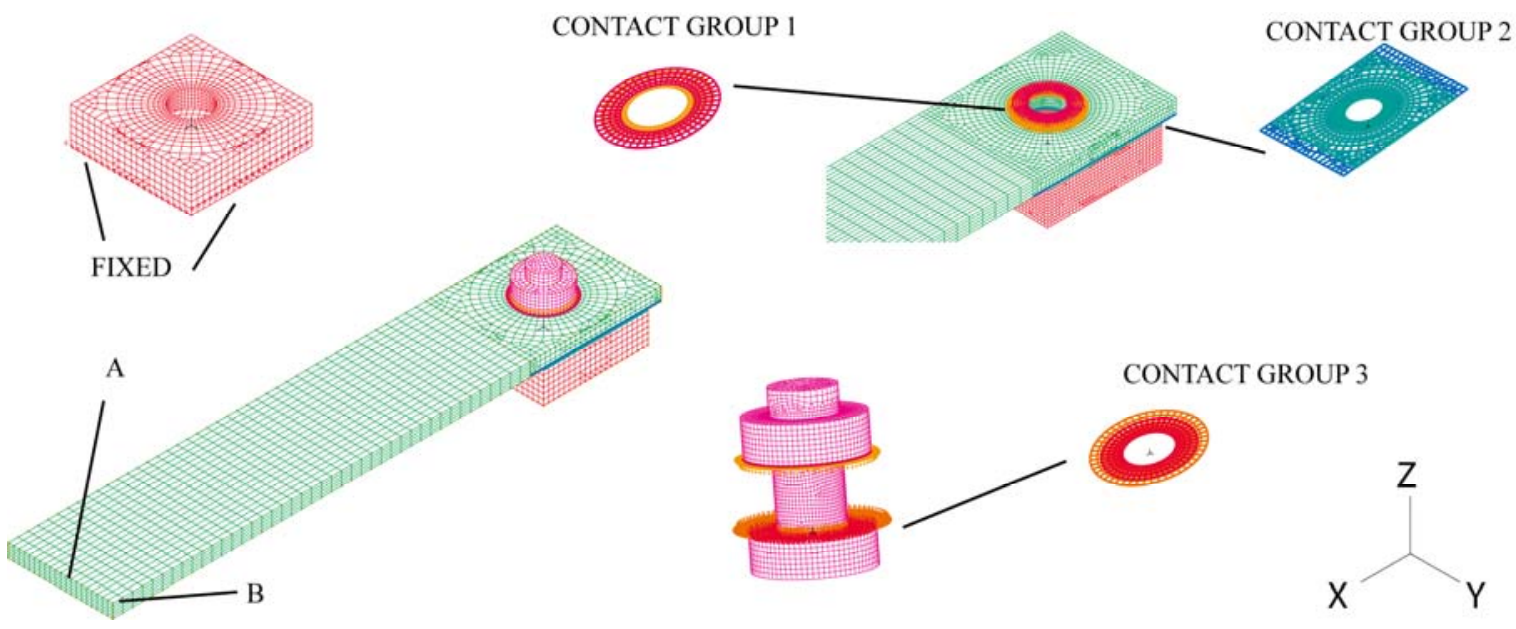

FIGURE I. MESHING CONDITIONS AND CONTACT GROUP DEFINE
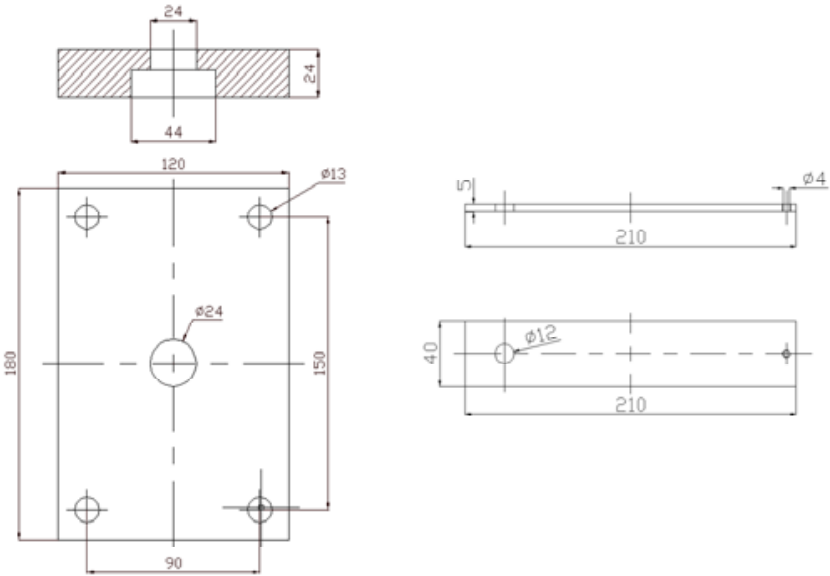

FIGURE II. DETAILS OF THE BOLTED JOINT CANTILEVER MODEL (IN MM)

TABLE I. SIMULATION PARAMETERS

\begin{tabular}{cccc}
\hline Material Density & Young Module & Poisson Ratio & $\begin{array}{c}\text { Bolt Preloading } \\
\text { Force }\end{array}$ \\
\hline $7800 \mathrm{~kg} / \mathrm{m}^{3}$ & $2.05 * 10^{11} \mathrm{~N} / \mathrm{m}^{2}$ & 0.3 & $100 \mathrm{~N}$ \\
\hline Solution Time & Solution Method & Steps & $\begin{array}{c}\text { Coulomb } \\
\text { Friction } \\
\text { Coefficient }\end{array}$ \\
\hline $0.6 \mathrm{~s}$ & Bathe & 1500 & 0.1 \\
\hline $\begin{array}{c}\text { Bolt Element } \\
\text { Number }\end{array}$ & $\begin{array}{c}\text { Beam Element } \\
\text { Number }\end{array}$ & $\begin{array}{c}\text { Base Element } \\
\text { Number }\end{array}$ & $\begin{array}{c}\text { Contact Force } \\
\text { Tolerance }\end{array}$ \\
\hline 41734 & 15417 & 21239 & 0.05 \\
\hline
\end{tabular}

IV. LINEAR RESONANT FREQUENCY CALCULATION

The simulation of modal and resonant frequencies of bolted joint connected structures can be analyzed by means of restarting through calculating the static response under preload of the bolt. But the changed of the bolt connection preloading force cannot influence the structural dynamics response characteristics via linear dynamics method. Therefore, it is necessary to analyze and calculate the influence of local nonlinearity on the dynamic response of the structure under the time-varying load. Dynamics response of bolted joint connection structure can be simulated by nonlinear finite element program through implicit calculation method by utilizing energy and displacement convergence principle. The time response of dynamic is carried out by constructing a linear sweep excitation force to analyze. The response results are sampled and analyzed by Fast Fourier Transform. Step-by-Step sinusoidal linear sweep load is constructed as follows:

$$
F(t)=F_{0} \sin \left(2 * \pi * \alpha t+2 * \pi * \frac{1}{2} \beta t^{2}\right)
$$

where $\alpha$ is the starting frequency; $\alpha+\beta$ means the cut-off frequency; $t$ is the calculating time. The sampling frequency is chosen according to twice the maximum frequency of the sample signal which means Nyquist Frequency. Frequency resolution using FFT is as following equation:

$$
f_{0}=f_{s} / N=1 / N T_{s}=1 / T
$$

Where is the number of sampling points, $f_{s}$ is the sampling frequency, $T_{s}$ means the sampling interval, $N T_{s}$ is the analogy signal before sampling the length of time. 

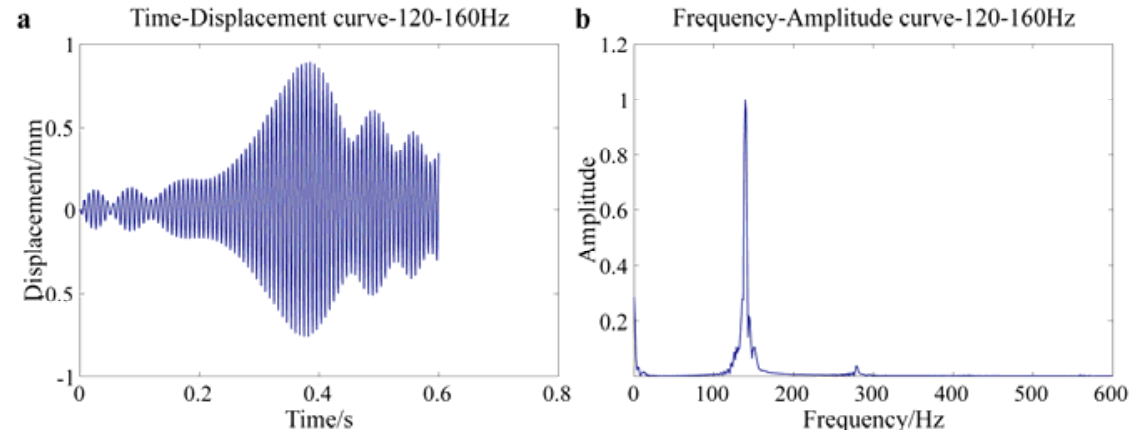

FIGURE III. (A) DISPLACEMENT-TIME CURVE OF POINT B UNDER SWEEP EXCITATION BETWEEN 120-160HZ (B) AMPLITUDE-FREQUENCY CHARACTERISTIC CURVE OF POINT B UNDER SWEEP EXCITATION BETWEEN 120-160HZ

The Sinusoidal swept load is applied at the excitation point A, with the starting and cut-off frequencies being $120 \mathrm{~Hz}$ and $160 \mathrm{~Hz}$. Sampling frequency is $5000 \mathrm{~Hz}$ and the calculating time is $0.6 \mathrm{~s}$. The frequency resolution is $1.67 \mathrm{~Hz}$. The mean value filtering is performed first when analysis the frequency domain result. Result of swept frequency analysis of the single cantilever beam is $f_{N}^{0}=139.20 \mathrm{~Hz}$. Figure 3 shows the results
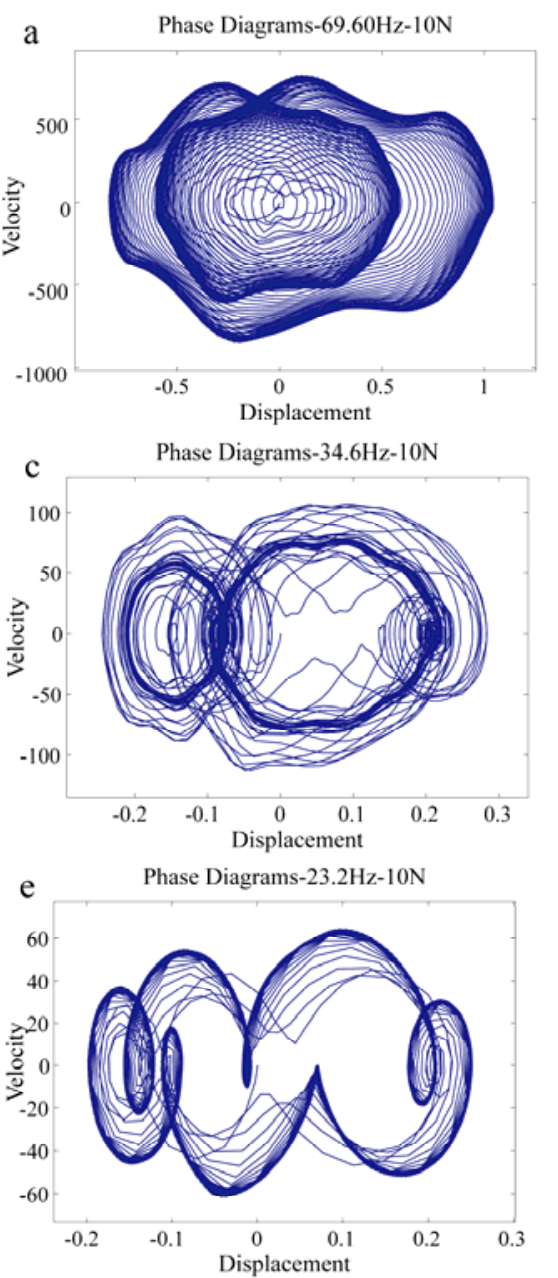

about the calculated time-displacement curve and Fast Fourier Transform of which. The result calculated above is more close to the actual dynamical characteristics when considering the influence of contact on the linear dynamic response of the structure.
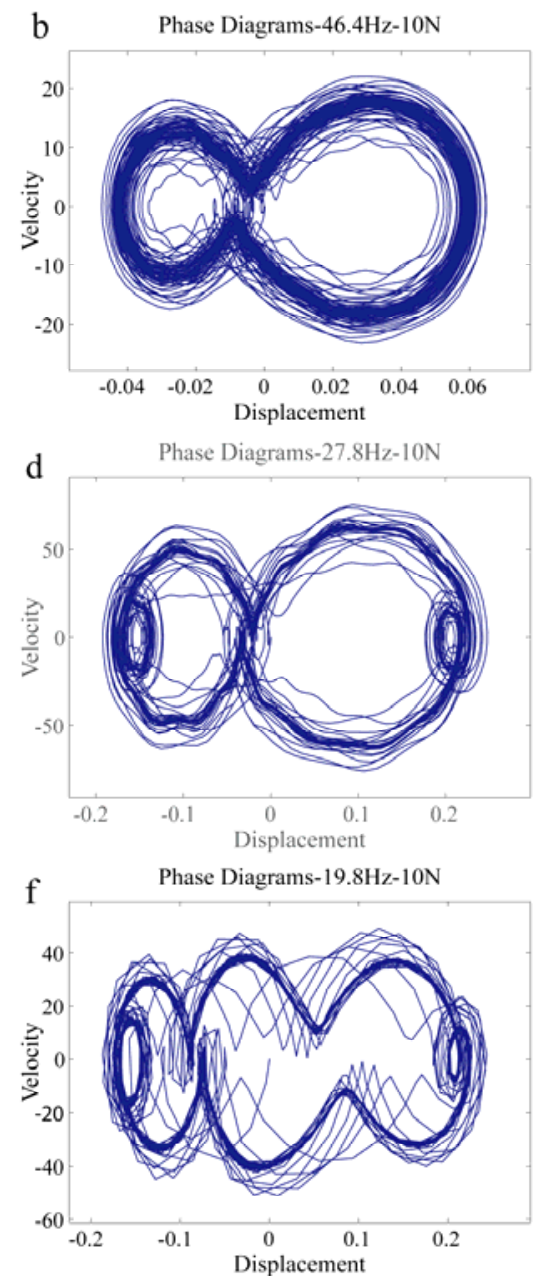

FIGURE IV. PHASE CURVE UNDER CONDITIONS $F_{N}=10 N \quad$ A) $f=f_{N}^{0} / 2$; в) $f=f_{N}^{0} / 3$;C) $f=f_{N}^{0} / 4$; D) $f=f_{N}^{0} / 5$; E) $f=f_{N}^{0} / 6$; F) $f=f_{N}^{0} / 7$ 


\section{Super Harmonic Resonance Response Phase DiAGRAM CALCULATION SIMULATION}

After calculated the dynamics response characteristic through method above, the time based load is applied on the excitation point $\mathrm{A}$. The load equation expressed as follows:

$$
F=F_{N} \sin (2 * \pi * f * t) \quad f=f_{N}^{0} / n, n=2,3, \ldots 7
$$

where $F_{N}=10 \mathrm{~N}, f$ is the frequency of the load frequency. The meshing condition and constraint are equal as part4. Phase diagrams are obtained through the calculation of the displacement response and the velocity response of the sampling point B. Figure 4 shows the phase space curve under sinusoidal excitation of different frequencies. The response of the structure under $1 / 2$ times of the first -order resonant frequency excitation is dominated by the free-vibration response which vibrated at frequency $f_{N}^{0}$. When the load amplitude is constant and the external load excitation frequency decreases to $1 / 3$ of the resonant frequency, the phase space curve has no bifurcation point and the response is not obvious at frequency $f_{N}^{0}$. The system response is quasiperiodic motion. When the frequency drops to $1 / 6$ of the resonant frequency, the space curve still presents the complex state. In addition, we can get the conclusion that superharmonic response generated when the excitation frequency is $1 / 2$ of the resonant frequency under strong nonlinearity conditions (structural response to divergence is strongly dependent on the energy convergence criterion and the convergence tendency of the strong nonlinear non-linear bolt connection structure). The system response is affected by the nonlinear term and the free vibration term with frequency $f_{N}^{0}$ is not attenuated. Under excitation of frequency at $f=f_{N}^{0} / n, n \neq 2$, the free vibration term existed and the amplitude of freevibration is smaller than that of the forced excitation.

\section{CONCLUSION}

The simulation in this paper is based on the principle of displacement and energy convergence. The nonlinear response is modeled and simulated. The super-harmonics response characteristics of the bolted joint connection structure under strong nonlinear conditions are obtained through nonlinear finite element analysis method. Result of the system is calculated under frequency. The linear dynamics characteristic analysis cannot fully reflect the structural dynamics characteristics when nonlinear boundary conditions exist on structure of the response result analysis of the calculation.

\section{REFERENCES}

[1] Čelič D, Boltežar M. Identification of the dynamic properties of joints using frequency-response functions[J]. Journal of Sound and Vibration, 2008, 317(1-2): 158-174

[2] Iwan W D. A Distributed-Element Model for Hysteresis and Its SteadyState Dynamic Response[J]. Journal of Applied Mechanics, 1966, 33(4): 893-\&.

[3] Crawley E F, Odonnell K J. Force-State Mapping Identification of Nonlinear Joints[J]. Aiaa Journal, 1987, 25(7): 1003-1010.
[4] Jalali H, Ahmadian H, Mottershead J E. Identification of nonlinear bolted lap-joint parameters by force-state mapping[J]. International Journal of Solids and Structures, 2007, 44(25-26): 8087-8105.

[5] Crawley E F, Aubert A C. Identification of Nonlinear Structural Elements by Force-State Mapping[J]. Aiaa Journal, 1986, 24(1): 155162

[6] Liu J, Chen X, Gao J, et al. Multiple-source multiple-harmonic active vibration control of variable section cylindrical structures: A numerical study[J]. Mechanical Systems and Signal Processing, 2016.

[7] Blachowski B, Gutkowski W. Effect of damaged circular flange-bolted connections on behaviour of tall towers, modelled by multilevel substructuring[J]. Engineering Structures, 2016, 111: 93-103.

[8] Abid M, Khan A, Nash D H, et al. Optimized bolt tightening strategies for gasketed flanged pipe joints of different sizes[J]. International Journal of Pressure Vessels and Piping, 2016.

[9] Zhu S, Shao G, Wang Y, et al. Mechanical behavior of the CFRP lattice core sandwich bolted splice joints[J]. Composites Part B: Engineering, 2016, 93: 265-272.

[10] K Worden G R T. Nonlinearity In Structural Dynamics Detection, Identification and Modelling[J], 2001. 